\title{
Fat crystal networks - structure and properties
}

Alejandro G. Marangoni

Dept. of Food Science, University of Guelph, Guelph, ON N1G2W1, Canada

Ph: (519) 824-4120, Fax: (519) 824-6631, e-mail: amarango@uoguelph.ca

When triglycerides are cooled from the melt to a temperature below their melting point, they undergo a liquid-solid transformation to form primary crystals with characteristic polymorphism. These crystals associate, or grow into each other, to form increasingly larger aggregates, which further interact, resulting in a continuous three-dimensional network. The macroscopic properties of a fat crystal network are affected by all these levels of structure, however, most directly by the level of structure closest to the macroscopic world. Most of the research in this area has been directed towards establishing a link between molecular structure and phase behavior, crystal habit, in particular crystal polymorphism, and the macroscopic properties of fats. Not including all levels of structure in the analysis is undoubtedly a major reason why these attempts have only been partially successful. In order to truly understand, and eventually predict, the macroscopic properties of fat-containing products, it is necessary to characterize and define the different levels of structure present in the material and their respective relationship to a macroscopic property. A macroscopic property is not always simply and directly related to molecular structure.

Work in our laboratory has shown that the rheological properties of edible fats are strongly dependent on the microstructure of their fat crystal networks using fractal scaling relationships and developed a particle counting method for the determination of the fractal dimension from polarized light micrographs (Narine and Marangoni, 1999. Physical Review E 59:1908). We have also developed a mechanical and structural model which relates the Young's modulus (E) of an isotropic material to particle properties, solids' volume fraction, and the spatial distribution of that mass (Marangoni, 2000. Phys. Rev B 62: 13951; Narine and Marangoni,1999. Physical Review E 60:6991), namely:

$\mathrm{E} \sim \frac{\mathrm{A}}{\pi \mathrm{a} \gamma \mathrm{d}_{\mathrm{o}}^{2}} \Phi^{\frac{1}{3-\mathrm{D}}}$

where A is Hamacker's constant, a corresponds to the diameter of primary crystal aggregates $(<5: m)$, ( to the strain imposed during the mechanical test, $d_{0}$ to the intercluster separation distance, $M$ to the volume fraction of solids, and $D$ to the fractal dimension of the network. Processing conditions (heat and mass transfer) can affect some, or all, of these parameters, particularly at the level of liquid structure. The possibility of structuring the solid state via manipulation of liquid structure will be explored in this presentation. 
Figure 1. Atomic force micrographs of cocoa butter crystallized at $22^{\circ} \mathrm{C}$ at different levels of magnification. Crystallite clustering into increasingly larger clusters is shown.
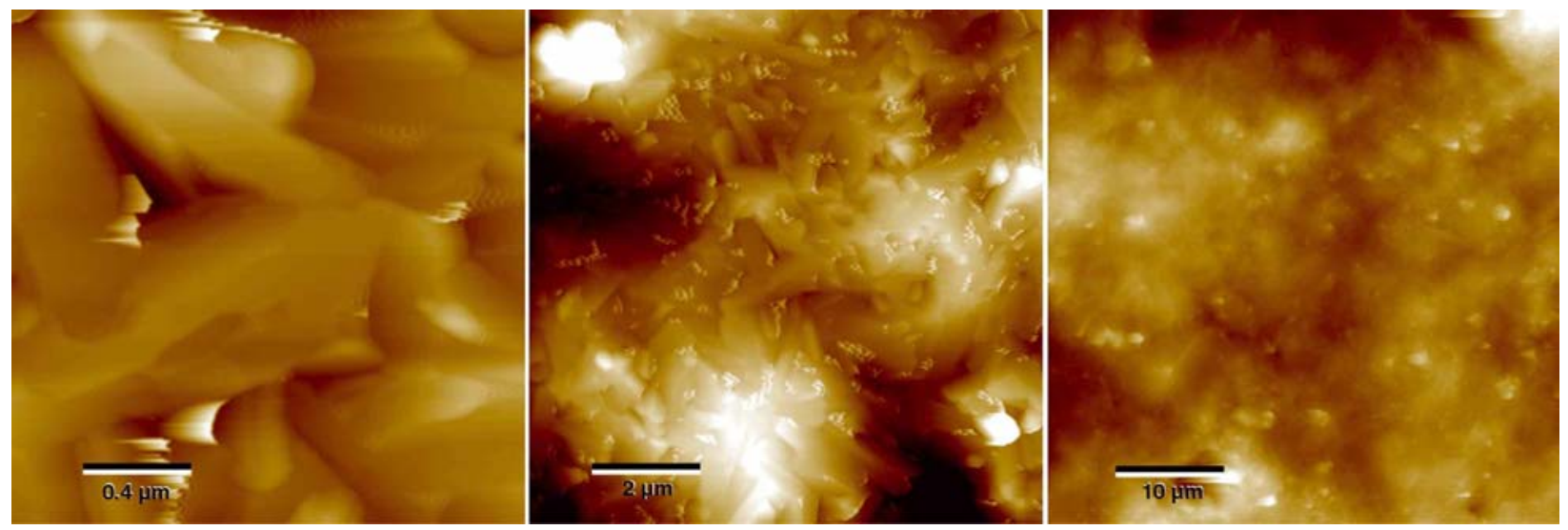